\title{
Regorafenib-induced exacerbation of chronic immune thrombocytopenic purpura in remission: A case report
}

\author{
SHIRO KIMBARA $^{1}$, YOSHINORI IMAMURA ${ }^{1}$, KIMIKAZU YAKUSHIJIN ${ }^{1}$, AKO HIGASHIME $^{1}$, \\ TAIJI KOYAMA ${ }^{1}$, YOSHIMI FUJISHIMA ${ }^{1}$, YOHEI FUNAKOSHI ${ }^{1}$, MASANORI TOYODA $^{1}$, \\ NAOMI KIYOTA ${ }^{1,2}$, HIROSHI MATSUOKA ${ }^{1}$ and HIRONOBU MINAMI ${ }^{1,2}$ \\ ${ }^{1}$ Department of Medical Oncology/Hematology; ${ }^{2}$ Cancer Center, Kobe University Hospital, Kobe, Hyogo 650-0017, Japan
}

Received December 27, 2019; Accepted November 12, 2020

DOI: $10.3892 / \mathrm{mco} .2020 .2192$

\begin{abstract}
Regorafenib is an oral multi-kinase inhibitor which targets tumor angiogenesis, the tumor microenvironment and oncogenesis. Based on this mode of action, regorafenib has a broad spectrum of toxicities. However, at present, few reports have focused on autoimmune adverse events. We report a first case of regorafenib-induced exacerbation of chronic immune thrombocytopenic purpura in remission during treatment for the patients with heavily treated advanced colorectal cancer. This case report highlights the need for caution with regard to regorafenib treatment in patients with cancer with concomitant immune thrombocytopenic purpura.
\end{abstract}

\section{Introduction}

Regorafenib is an oral multi-kinase inhibitor which targets tumor angiogenesis [vascular endothelial growth factor receptor (VEGFR)1-3 and TIE2], tumor microenvironment (platelet-derived growth factor receptor $\beta$ (PDGFR $\beta$ ) and fibroblast growth factor receptor-1), and oncogenesis (c-KIT, RET, RAF-1 and B-RAF) (1). Based on this mode of action, regorafenib has a broad spectrum of toxicities (2). To date, however, few reports have focused on autoimmune adverse events.

\section{Case report}

The patient was a Japanese woman who had a past medical history of chronic immune thrombocytopenic purpura (ITP) which developed at the age of 38 years and was treated with steroid therapy, which resulted in remission for more than 20 years without medication. She was diagnosed with recurrent

Correspondence to: Dr Yoshinori Imamura, Department of Medical Oncology/Hematology, Kobe University Hospital, 7-5-2 Kusunoki-cho, Chuo-ku, Kobe, Hyogo 650-0017, Japan E-mail: yimamura@med.kobe-u.ac.jp

Key words: regorafenib, multi-kinase inhibitor, platelet-derived growth factor receptor beta, chronic immune thrombocytopenic purpura, colorectal cancer colon cancer at age 66 years, after primary surgery and adjuvant chemotherapy with capecitabine plus oxaliplatin. She had received three lines of palliative chemotherapy including fluorouracil, leucovorin, and irinotecan (FOLFIRI) plus bevacizumab, panitumumab monotherapy and trifluridine/tipiracil. Platelet-associated IgG (PAIgG) was not detected when FOLFIRI plus bevacizumab was initiated. High-grade thrombocytopenia was not observed during treatment for recurrent colon cancer.

As a standard therapy in the late-line setting for recurrent/ metastatic colorectal cancer $(3,4)$, treatment with regorafenib $160 \mathrm{mg}$ orally once daily for 21 days on/7-days off in a 28-day cycle was initiated at the age of 68 years. Platelet count was $167 \times 10^{9} / 1$ on day 1 but dropped to $61 \times 10^{9} / 1$ on day 15 , and regorafenib was continued. On day 18 , she vomited blood and presented at the emergency department. Laboratory examination showed severe thrombocytopenia with a platelet count of $5 \times 10^{9} / 1$ (Table I). Petechiae and purpura in the extremities and hemorrhagic blisters in the oral mucosa were also observed (Fig. 1A). Multiple platelet transfusions were given, but the response was poor. Further laboratory examination showed increased PAIgG of $176 \mathrm{ng}$ (normal range $<27.6 \mathrm{ng}$ ) and negative $\mathrm{IgG}$ for $H$. pylori and heparin-induced thrombocytopenia antibody (Table I). There were no clinical manifestations suggested systemic lupus erythematosus (SLE), such as arthritis, mucocutaneous involvement or Raynaud's phenomenon. Diagnostic criteria of SLE were not met. Bone marrow examination revealed normal hematopoiesis, slightly increased megakaryocytes and no myelodysplasia or tumor metastasis (Fig. 1B). There was no evidence of other risk factors for exacerbation of ITP, including a history of taking any dietary supplements or medications, or viral infections. Taken together, these findings strongly suggested regorafenib exacerbated ITP. Regorafenib was permanently discontinued, and prednisone $1 \mathrm{mg} / \mathrm{kg} /$ day was administrated on day 21 . The hemorrhagic diathesis resolved one week later, and the severe thrombocytopenia gradually recovered (Fig. 1C).

\section{Discussion}

Thrombocytopenia associated with regorafenib is not rare. A meta-analysis reported incidences of all-grade and high-grade 
Table I. Laboratory data.

\begin{tabular}{|c|c|c|}
\hline Variable & Reference range & Result \\
\hline White blood cell count, $100 / \mu 1$ & $33-86$ & 50 \\
\hline Red blood cell count, $10^{6} / \mu 1$ & $3.86-4.92$ & 2.90 \\
\hline Hemoglobin, g/dl & $11.6-14.8$ & 9.3 \\
\hline Hematocrit, \% & $35.1-44.4$ & 28.4 \\
\hline Platelet count, $10^{9} / 1$ & $158-348$ & 5 \\
\hline Immature platelet fraction, $\%$ & $1-4.8$ & 16.3 \\
\hline APTT, sec & $25.0-38.0$ & 31.6 \\
\hline PT, $\%$ & $70.0-130.0$ & 94.0 \\
\hline Fibrinogen, mg/dl & $200-400$ & 298 \\
\hline $\mathrm{D}$ dimer, $\mu \mathrm{g} / \mathrm{ml}$ & $<1$ & 4.1 \\
\hline Lactate dehydrogenase, U/l & $124-222$ & 615 \\
\hline Aspartate transaminase, $\mathrm{U} / \mathrm{l}$ & $13-30$ & 32 \\
\hline Alanine aminotransferase, U/1 & $7-23$ & 17 \\
\hline Total bilirubin, $\mathrm{mg} / \mathrm{ml}$ & $0.4-1.5$ & 1.6 \\
\hline Creatinine, mg/dl & $0.46-0.79$ & 0.87 \\
\hline Blood urea nitrogen, mg/dl & $8-20$ & 26.8 \\
\hline C-reactive protein, $\mathrm{mg} / \mathrm{dl}$ & $0.00-0.14$ & 1.55 \\
\hline PA IgG, ng/10 7 cells & $<27.6$ & 176.8 \\
\hline $50 \%$ complement hemolysis, $\mathrm{U} / \mathrm{ml}$ & $25-51$ & 30.2 \\
\hline Complement C3, mg/dl & $73-138$ & 72 \\
\hline Complement $\mathrm{C} 4, \mathrm{mg} / \mathrm{dl}$ & $11-31$ & 11 \\
\hline Antinuclear antibody, IF & $<40$ & $320 x$ \\
\hline Antinuclear antibody pattern & & Centromere pattern \\
\hline HIT antibody, U/ml & $<1$ & $<0.6$ \\
\hline IgG antibody for $H$. pylori, $\mathrm{U} / \mathrm{ml}$ & $<10$ & 3 \\
\hline Hepatitis B surface antigen, IU/ml & $<0.0049$ & $<0.003$ \\
\hline Hepatitis $\mathrm{C}$ virus antibodies, COI & $<0.99$ & 0.04 \\
\hline $\mathrm{HIV}$ antibody/antigen combo assay, $\mathrm{S} / \mathrm{CO}$ & $<0.99$ & 0.12 \\
\hline
\end{tabular}

APTT, activated partial thromboplastin time; COI, cutoff index; HIT, heparin-induced thrombocytopenia; HIV, human immunodeficiency virus; $\mathrm{IF}$, indirect immunofluorescence; $\mathrm{MCH}$, mean corpuscular hemoglobin; $\mathrm{MCHC}$, mean corpuscular hemoglobin concentration; MCV, mean corpuscular volume; PA IgG, platelet-associated IgG; PT, prothrombin time; S/CO, signal-to-cutoff.

thrombocytopenia 22 and 3\%, respectively (5). Inhibition of VEGFR is a potential mechanism of regorafenib-induced myelosuppression $(6,7)$. Conventional thrombocytopenia is associated with bone marrow hypoplasia and responds to blood transfusion. In the present case, in contrast, normal hematopoiesis was maintained, and thrombocytopenia was refractory to platelet transfusion, which is likely explained by an autoimmune mechanism.

Diagnosis of ITP requires exclusion of a variety of potential causes for thrombocytopenia. Many conditions which cause decreased platelet production such as bone marrow damage, infiltration and replacement of the bone marrow due to malignancies and myelodysplastic syndromes were excluded by findings form the bone marrow biopsy in the present case. Drug-induced thrombocytopenia (DITP) is difficult to be distinguished from ITP. However, a history of ITP and unrecovered thrombocytopenia after discontinuation of regorafenib suggested more likely ITP than DITP (8). Moreover, a very low platelet count nadir less than 20x10 $/ 1$, response to steroid and a positive anti-platelet autoantibody test are supposed to help precise diagnosis of ITP from expert opinions $(9,10)$. Based on the above, the diagnosis of ITP was very likely.

ITP is an autoimmune disease which is characterized by platelet destruction associated with antibodies to platelets and megakaryocyte dysfunction (11). The pathogenesis of ITP is complicated and has not been fully clarified. Recent findings suggest that dysfunction of mesenchymal stem cells (MSCs) plays an important role $(12,13)$. MSCs derived from ITP patients (MSCs-ITP) showed impaired self-proliferative capacity and the loss of immunosuppressive function. Interestingly, treatment of MSCs-ITP with PDGF-BB, a ligand of PDGFR $\beta$, could reverse the defect of MSC-ITP in vitro (13). In this basis, regorafenib-induced inhibition of PDGF-BB/PDGFR $\beta$ signaling might trigger dysfunction of MSCs, resulting in the exacerbation of ITP. VEGF/VEGFR signaling is another important target of regorafenib, however, exacerbation of ITP had not occurred during bevacizumab 

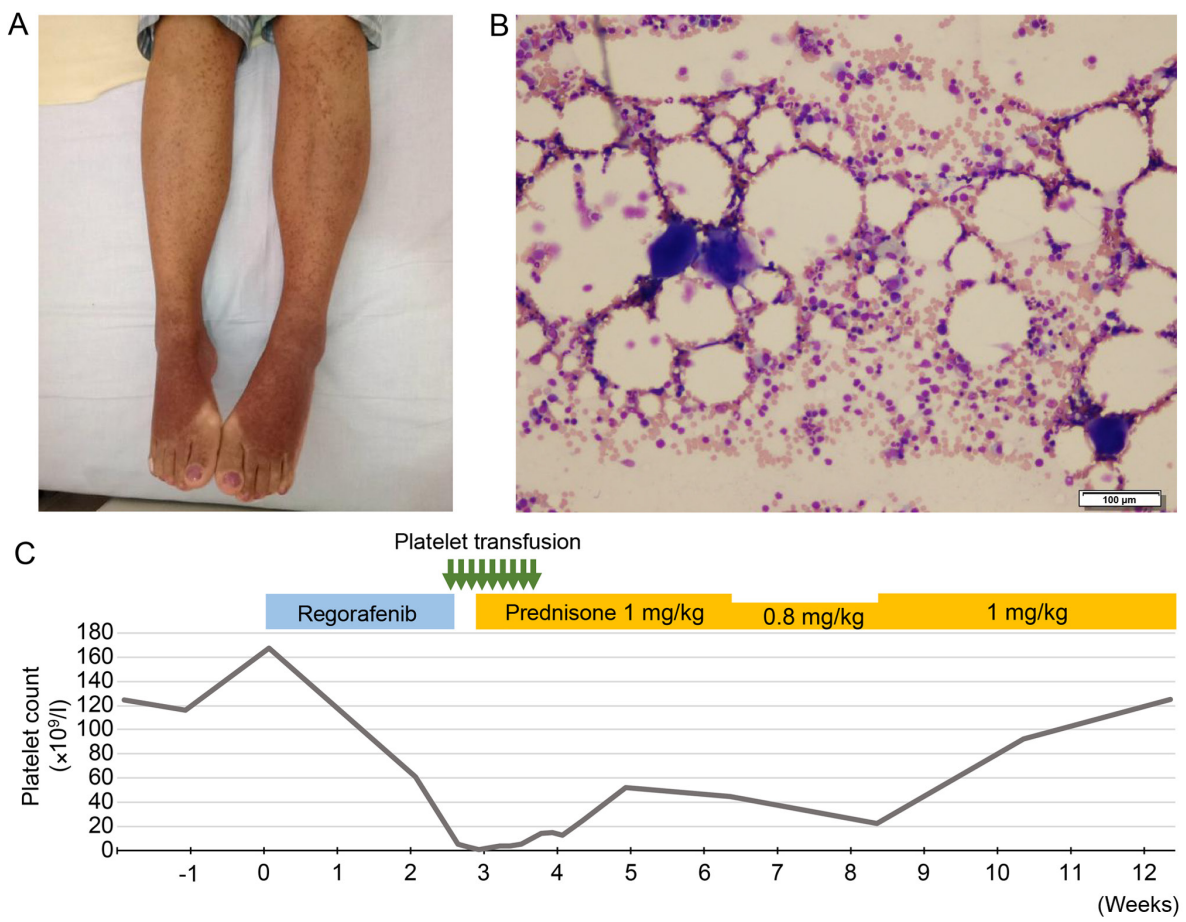

Figure 1. Clinical and pathological findings. (A) Petechiae and purpura in the extremities on day 21. (B) Histopathologic findings of bone marrow examination. Trilineage hematopoiesis was maintained. The megakaryocyte count was slightly increased. Myelodysplasia or tumor metastasis were not observed. Scale bar, $100 \mu \mathrm{m}$. (C) Clinical course of treatment with regorafenib and immune thrombocytopenic purpura. Green arrows indicate 10 units of platelet transfusion. Prednisone ( $1 \mathrm{mg} / \mathrm{kg} /$ day) was administered from day 21 and reduced to $0.8 \mathrm{mg} / \mathrm{kg} / \mathrm{day}$ on day 45 . As the platelet count decreased, prednisone was again increased to $1 \mathrm{mg} / \mathrm{kg} / \mathrm{day}$ on day 59 .

containing treatment in the first-line setting at age of 66 years, which supports the hypothesis above.

Several multi-kinase inhibitors other than regorafenib also inhibit PDGF/PDGFR signaling, which may exacerbate ITP. Imatinib and sunitinib have been reported to induce immune thrombocytopenia $(14,15)$, albeit that these studies did not investigate the possibility of pre-existing MSC dysfunction.

In conclusion, we report the first case of regorafenib-induced exacerbation of ITP in remission. This case report highlights the need for caution with regard to regorafenib treatment in cancer patients with concomitant ITP.

\section{Acknowledgements}

The authors would like to thank Dr Maki Kanzawa (Department of Diagnostic Pathology, Kobe University Graduate School of Medicine, Kobe, Japan) for pathological diagnosis.

\section{Funding}

No funding was received.

\section{Availability of data and materials}

The datasets used and/or analyzed during the current study are available from the corresponding author on reasonable request.

\section{Authors' contributions}

SK and YI made substantial contributions to the conception and design of the study. SK, YI, KY, AH and NK made substantial contributions to the acquisition of the data. SK, YI and KY drafted the manuscript. AH, TK, YFuj, YFun, MT, NK, HMa and HMi made substantial contributions to the analysis and interpretation of the data and were involved in revising the manuscript critically for important intellectual content. All authors read and approved the final manuscript.

\section{Ethics approval and consent to participate}

Not applicable.

\section{Patient consent for publication}

Written informed consent was obtained from the patient for publication of the clinical data and images.

\section{Competing interests}

The authors declare that they have no competing interests.

\section{References}

1. Wilhelm SM, Dumas J, Adnane L, Lynch M, Carter CA, Schütz G, Thierauch KH and Zopf D: Regorafenib (BAY 73-4506): A new oral multikinase inhibitor of angiogenic, stromal and oncogenic receptor tyrosine kinases with potent preclinical antitumor activity. Int J Cancer 129: 245-255, 2011.

2. Krishnamoorthy SK, Relias V, Sebastian S, Jayaraman V and Saif MW: Management of regorafenib-related toxicities: A review. Therap Adv Gastroenterol 8: 285-297, 2015. 
3. Grothey A, Van Cutsem E, Sobrero A, Siena S, Falcone A, Ychou M, Humblet Y, Bouché O, Mineur L, Barone C, et al: Regorafenib monotherapy for previously treated metastatic colorectal cancer (CORRECT): An international, multicentre, randomised, placebo-controlled, phase 3 trial. Lancet 381: 303-312, 2013.

4. Li J, Qin S, Xu R, Yau TC, Ma B, Pan H, Xu J, Bai Y, Chi Y, Wang L, et al: Regorafenib plus best supportive care versus placebo plus best supportive care in Asian patients with previously treated metastatic colorectal cancer (CONCUR): A randomised, double-blind, placebo-controlled, phase 3 trial. Lancet Oncol 16: 619-629, 2015.

5. Zhao B and Zhao H: Incidence and risk of hematologic toxicities in cancer patients treated with regorafenib. Oncotarget 8: 93813-93824, 2017.

6. Gerber HP, Malik AK, Solar GP, Sherman D, Liang XH, Meng G, Hong K, Marsters JC and Ferrara N: VEGF regulates haematopoietic stem cell survival by an internal autocrine loop mechanism. Nature 417: 954-958, 2002.

7. Hattori K, Heissig B, Wu Y, Dias S, Tejada R, Ferris B, Hicklin DJ, Zhu Z, Bohlen P, Witte L, et al: Placental growth factor reconstitutes hematopoiesis by recruiting VEGFR1(+) stem cells from bone-marrow microenvironment. Nat Med 8 : $841-849,2002$

8. George JN and Aster RH: Drug-induced thrombocytopenia: Pathogenesis, evaluation, and management. Hematology Am Soc Hematol Educ Program 153-158, 2009.

9. Salib M, Clayden R, Clare R, Wang G, Warkentin TE, Crowther MA, Lim W, Nazi I, Kelton JG and Arnold DM: Difficulties in establishing the diagnosis of immune thrombocytopenia: An agreement study. Am J Hematol 91: E327-E329, 2016.

10. Kelton JG, Vrbensky JR and Arnold DM: How do we diagnose immune thrombocytopenia in 2018? Hematology Am Soc Hematol Educ Program 2018: 561-567, 2018.
11. McMillan R, Wang L, Tomer A, Nichol $\mathrm{J}$ and Pistillo $\mathrm{J}$ : Suppression of in vitro megakaryocyte production by antiplatelet autoantibodies from adult patients with chronic ITP. Blood 103: 1364-1369, 2004

12. Zhang D, Li H, Ma L, Zhang X, Xue F, Zhou Z, Chi Y, Liu X, Huang Y, Yang Y and Yang R: The defective bone marrow-derived mesenchymal stem cells in patients with chronic immune thrombocytopenia. Autoimmunity 47: 519-529, 2014

13. Zhang JM, Feng FE, Wang QM, Zhu XL, Fu HX, Xu LP, Liu KY, Huang XJ and Zhang XH: Platelet-derived growth factor-BB protects mesenchymal stem cells (MSCs) derived from immune thrombocytopenia patients against apoptosis and senescence and maintains MSC-mediated immunosuppression. Stem Cells Transl Med 5: 1631-1643, 2016.

14. Radwi M and Cserti-Gazdewich C: Drug-induced immune thrombocytopenia associated with use of tyrosine kinase inhibitor imatinib. J Taibah University Med Sci 10: 365-368, 2015.

15. Ansari Z and George MK: Drug-induced immune-mediated thrombocytopenia secondary to sunitinib in a patient with metastatic renal cell carcinoma: A case report. J Med Case Rep 7: 54, 2013.

This work is licensed under a Creative Commons Attribution-NonCommercial-NoDerivatives 4.0 International (CC BY-NC-ND 4.0) License. 